\title{
LARYNGEAL ELECTROMYOGRAPHY IS HELPFUL FOR CARDIOVOCAL SYNDROME
}

\author{
Sevtap Akbulut ${ }^{1}$, Rahsan Inan', Mehmet Gökhan Demir ${ }^{3}$, Dogan Cakan ${ }^{1}$
}

Department of Otolaryngology Head and Neck Surgery, Dr. Lutfi Kirdar Kartal Training and Research Hospital, Istanbul, Turkey $^{1}$; Department of Neurology, Dr. Lutfi Kirdar Kartal Training and Research Hospital, Istanbul, Turkey²; Department of Otolaryngology Head and Neck Surgery, Etimesgut State Hospital, Ankara,Turkey ${ }^{3}$

Summary: Laryngeal electromyography is used in the evaluation of vocal cord paralysis to confirm the diagnosis, to guide the diagnostic work-up for etiology, to provide prognostic information and to help choose the correct treatment for the patient. Cardiovocal syndrome is characterised by vocal cord paralysis due to a cardiovascular disease. A wide spectrum of conditions can result in this syndrome. Here we present a case of cardiovocal syndrome in association with primary pulmonary hypertension. Laryngeal electromyography was used to guide the work-up of differential diagnosis and also for further intervention with respect to vocal cord paralysis in this patient.

Keywords: Laryngeal electromyography; Vocal cord paralysis; Cardiovocal syndrome; Ortner's syndrome;

Hoarseness

\section{Introduction}

Vocal cord paralysis represents a common neurolaryngological problem. It results in a breathy dysphonia with immobility of the ipsilateral vocal cord. Laryngeal electromyography (LEMG) is used in the evaluation of vocal cord paralysis. Information gathered from LEMG allows clinicians not only to confirm the diagnosis, but also to make the office work-up easier for clarifying the etiology $(1,2)$. In addition, it is well documented that LEMG can help to determine the prognosis of vocal cord paralysis (1, $3,4)$.

Cardiovocal syndrome, also known as Ortner's syndrome, is a rare condition in which cardiovascular pathology is the underlying cause of hoarseness $(5,6)$. Although it was initially associated with mitral stenosis, various clinical conditions such as pulmonary hypertension, enlarged pulmonary artery, thoracic aortic aneurysms and aberrant subclavian artery can result in recurrent laryngeal nerve (RLN) paralysis (7). Additionally, similar compression mechanisms can be detected on aorta causing dysphagia aortica and on subclavian artery causing dysphagia lusoria $(8,9)$.

Here we present a case of cardiovocal syndrome in association with primary pulmonary hypertension. LEMG was used to guide the work-up of differential diagnosis and also for further intervention regarding vocal cord paralysis in this patient.

\section{Case report}

A 34-year-old woman presented to the Department of ENT and Head \& Neck Surgery with a 2 months history of hoarseness that developed gradually. She also reported shortness of breath and dyspnea during exercise for the last couple of years. She did not mention any problem with swallowing but she aspirated occasionally. She was never a smoker. Voice Handicap Index-10 score was 32 (This is a patient-based self assessment voice symptom index on a scale from 0 to 40 (10)). Endoscopic laryngeal examination demonstrated left vocal cord paralysis and supraglottic compression (Figure 1a and $\mathrm{b}$ ). Maximum phonation time was 8 seconds.

LEMG revealed a large amount of positive sharp waves and fibrillation potentials with markedly reduced recruitment of polyphasic motor unit action potentials at the left thyroarytenoid muscle (Figure 2a and b). However, the right thyroarytenoid muscle and both cricothyroid muscles were evaluated as normal with normal waveforms and a full interference pattern with an absence of any spontaneous activity. These findings were consistent with subacute partial axonal loss (palsy) of the left RLN.

On the basis of this data, investigation for etiology of the left RLN paralysis focused on the lower neck and upper chest. Ultrasonography of her neck was negative for any mass or lymphadenopathy. She consulted firstly an internist and then a cardiologist. Echocardiography revealed dilated right atrium and ventricle, distended pulmonary arteries 


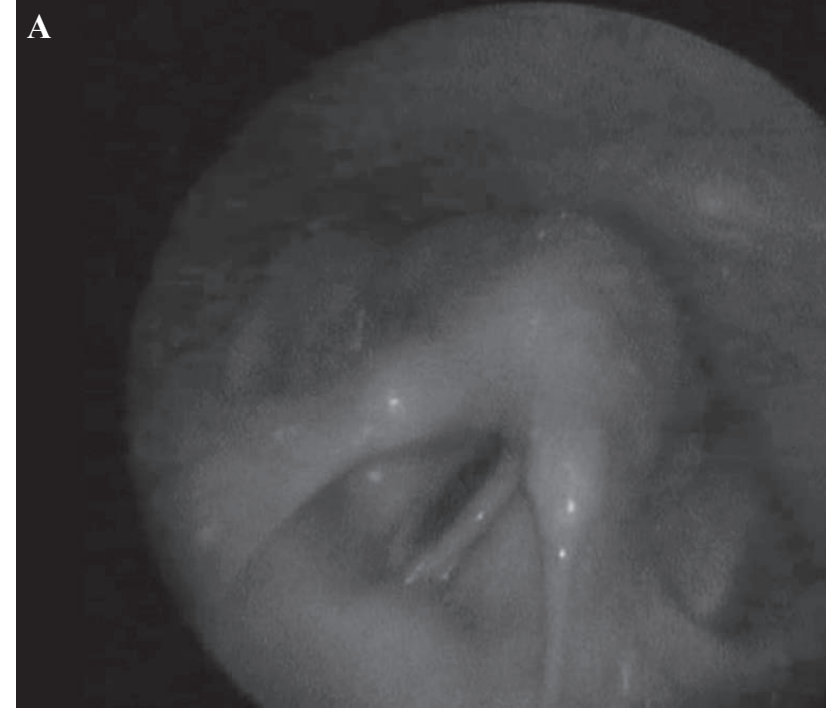

B

Fig. 1: Rigid endoscopic laryngeal examination showing left vocal cord paralysis. (a) During deep inspiration. (b) During phonation of $/ i /$ vowel.

(main pulmonary trunk $30.8 \mathrm{~mm}$, right pulmonary artery $23.4 \mathrm{~mm}$ and the left pulmonary artery $22 \mathrm{~mm}$ ) with severe pulmonary hypertension (pulmonary artery systolic pressure $103 \mathrm{~mm} \mathrm{Hg}$ ). Mild pulmonary insufficiency and moderate tricuspid insufficiency were also noted. Signs of bilateral small airway disease and atelectasis of left upper lobe were noted in computer tomography (CT) scan images. CT pulmonary arteriography demonstrated distended pulmonary arteries compressing the arch of aorta (Figure 3).

The patient was diagnosed with primary pulmonary hypertension. Paralysis of the left RLN resulted from the distended pulmonary artery. In-office Radiesse ${ }^{\circledR}$ Voice (Merz Pharma $\mathrm{GmbH} \& \mathrm{Co}$. KGaA, USA) injection was done to the left vocal cord of the patient through the cricothyroid
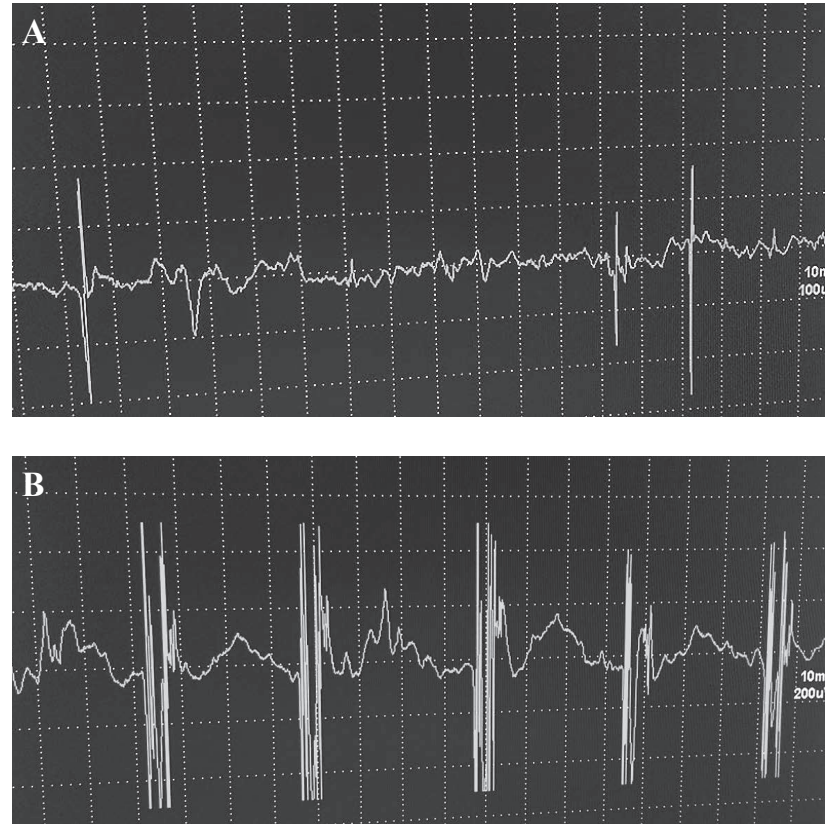

Fig. 2: (a) Fibrillation potentials on laryngeal electromyography. (Recorded from left thyroarytenoid muscle) (Sweep speed $10 \mathrm{~ms} /$ div, sensitivity $100 \mu \mathrm{V} /$ div). (b) Markedly decreased recruitment pattern (single-fiber oscillation) on laryngeal electromyography (Recorded from left thyroarytenoid muscle) (Sweep speed $10 \mathrm{~ms} /$ div, sensitivity $200 \mu \mathrm{V} / \mathrm{div})$.

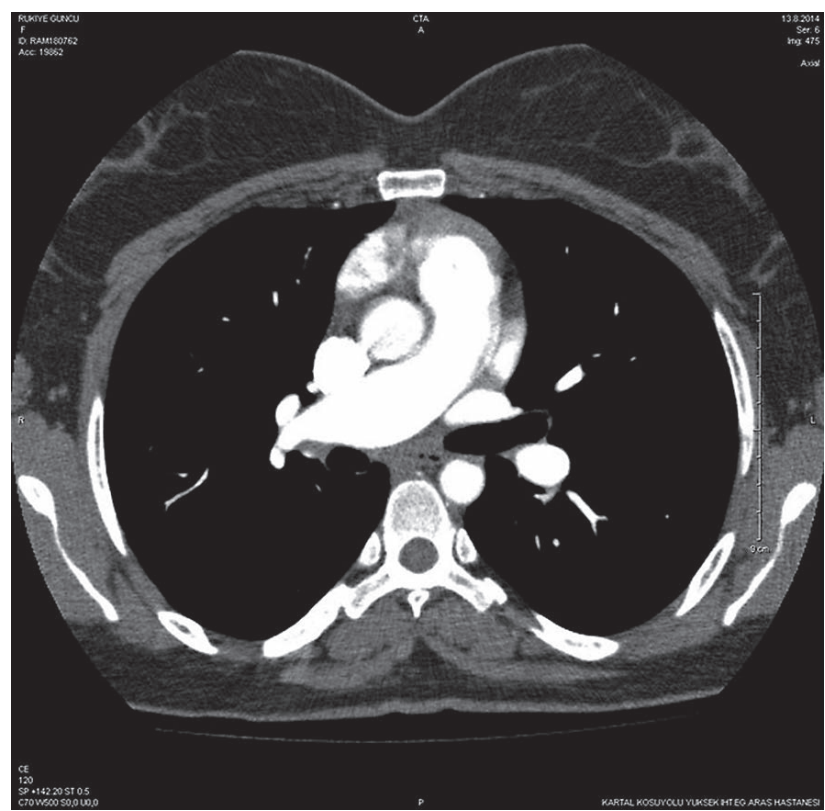

Fig. 3: CT pulmonary arteriography, axial view showing distended pulmonary arteries compressing the arch of aorta.

membrane. Complete glottic closure was achieved after the injection. Maximum phonation time was 18 seconds and Voice Handicap Index-10 score was 15 at a postoperative 4 weeks-follow-up visit. 


\section{Discussion}

Vocal cord paralysis must be investigated systematically. After taking a detailed history, an endoscopic laryngeal examination must be done with a flexible or rigid laryngoscope. There is no pathognomonic laryngeal position that indicates where the vagal nerve is injured in patients with vocal cord paralysis. Investigation to identify the precise cause should be done through detailed chest, neck, skull base and brain examinations.

LEMG helps to differentiate between superior laryngeal nerve palsy, RLN palsy and high vagal nerve palsy in vocal cord paralysis. The RLN controls all the muscles of the larynx except the cricothyroid. LEMG findings consistent with denervation or denervation and reinnervation in the thyroarytenoid muscle with normal LEMG findings in the cricothyroid muscle would indicate RLN involvement alone. This should direct the diagnostic work-up in the first place towards the chest and neck sites (1). In suspected patients, transthoracic echocardiography is performed to eliminate a cardiovascular cause. As a matter of fact, in our patient LEMG findings of denervation and reinnervation in the left thyroarytenoid muscle demonstrated isolated left RLN paralysis which later was shown to have resulted from a cardiovascular pathology.

The left RLN is more prone to injury by compression or traction because of its lengthy course, especially around the aortic arch and also its close relationship with major blood vessels in the mediastinum. The association of left RLN paralysis with a cardiovascular pathology was termed as cardiovocal syndrome $(5,6,11,12)$. It was first described in a series of three cases of mitral stenosis suffering from hoarseness by Ortner in 1897 (5). A wide variety of diseases, including congenital or adult cardiac conditions (such as mitral valve disorders, aneurysms of aorta and pulmonary artery, pulmonary hypertension, and tumors of left atrium and ventricles) and iatrogenic conditions (such as cardiovascular surgery and cardiac interventions) can result in hoarseness through RLN paralysis by either compressing or stretching effects $(6,11,12)$. It was hypothesized that palsy results from compression of the left RLN between the pulmonary artery and either the aorta or aortic ligament due to cardiopulmonary pathology (6). In the present case, a dilated pulmonary artery due to primary pulmonary hypertension was the reason for the RLN paralysis.

LEMG is also used to determine the neurophysiological state of laryngeal paralysis, to provide prognostic information, and consequently to decide the correct treatment $(1,2$, 4). The current evidence indicates that LEMG has a high specificity and positive predictive value, making it a reliable examination when abnormal findings are obtained. Abnormal findings, such as fibrillation potentials, positive sharp waves, and absent or reduced recruitment, predict a poor functional prognosis in vocal cord paralysis (4). However, when normal results are obtained using LEMG, it should be remembered that the sensitivity and negative predictive value are low for this test (3). In other words, poor prognosis is determined more accurately by LEMG, while one should be cautious when LEMG suggests good prognosis.

Treatment alternatives for vocal cord paralysis include observation, voice therapy and surgery such as injection laryngoplasty (temporary or permanent), medialization thyroplasty and reinnervation procedures (13). The decision regarding treatment is guided by the symptoms of the patient and expectations for recovery from the paralysis. Prognostic information provided with LEMG will help in this. It is recommended that patients with a clearly denervated larynx, i.e. those with spontaneous irritable muscles with fibrillation potentials, positive sharp waves, and complex repetitive discharges, will be good candidates for permanent procedures such as medialization laryngoplasty and, if necessary arytenoid adduction (1).

As our patient was severely hoarse and LEMG showed abnormal findings predicting a poor prognosis, a permanent injection laryngoplasty was performed in the office under local anesthesia.

\section{Conclusion}

Vocal cord paralysis is a common clinical condition; laryngeal electromyography (LEMG) is an extremely helpful test not only for guiding the diagnostic work-up but also for providing prognostic information in these patients. Cardiovocal syndrome is characterised by vocal cord paralysis due to a cardiovascular disease and can result from a variety of pathologies. Although rare, this syndrome should be kept in mind in differential diagnosis of vocal cord paralysis.

\section{Acknowledgements}

The authors would like to thank Daniel B. Magraw from Johns Hopkins University for editorial assistance in the preparation of this manuscript.

\section{References}

1. Woo P. Laryngeal electromyography is a cost-effective clinically useful tool in the evaluation of vocal fold function. Arch Otolaryngol Head Neck Surg 1998 Apr; 124(4): 472-475.

2. Ingle JW, Young VN, Smith LJ, Munin MC, Rosen CA. Prospective evaluation of the clinical utility of laryngeal electromyography. Laryngoscope 2014; 124 $2745-2749$.

3. Gavazzoni FB, Scola RH, Lorenzoni PJ, Kay CS, Werneck LC. The clinical value of laryngeal electromyography in laryngeal immobility. J Clin Neurosci 2011; 18(4): 524-527.

4. Rickert SM, Childs LF, Carey BT, Murry T, Sulica L. Laryngeal electromyography for prognosis of vocal fold palsy: a meta-analysis. Laryngoscope 2012; 122 : $158-161$.

5. Ortner N. Recurrent laryngeal nerve paralysis due to mitral valve stenosis. Wien Klin Wochenschr 1897; 10: 753-755.

6. Mulpuru SK, Vasavada BC, Punukollu GK, Patel AG. Cardiovocal syndrome: a systematic review. Heart Lung Circ 2008 Feb; 17(1): 1-4.

7. Verbeke X, Vliebergh J, Sauer M, Leys M. Hoarseness revealing Ortner's syndrome. Acta Clin Berg 2015; 70(3): 230

8. Abdul Haziz SR, Bickle I, Chong VH. Dysphagia aortica: a rare cause of dysphagia. BMJ Case Rep.

9. Reynolds I, McGarry J, Mullett H. Aberrant right retroesophageal subclavian artery causing esophageal compression. Clin Case Rep 2015 Oct; 3(10): 897-898. 
10. Rosen CA, Lee AS, Osborne J, Zullo T, Murry T. Development and validation of the voice handicap index-10. Laryngoscope 2004; 114(9): 1549-1556.

11. Subramaniam V, Herle A, Mohammmed N, Thahir M. Ortner's syndrome: case series and literature review. Braz J Otorhinolaryngol 2011 Sep-Oct; 77(5): 559-562. 12. Yuan SM. Hoarseness subsequent to cardiovascular surgery, intervention, maneu- ver and endotracheal intubation: the so-called iatrogenic Ortner's (cardiovocal) syndrome. Cardiol J 2012; 19(6): 560-566.

13 Misono S, Merati AL. Evidence-based practice: evaluation and management of unilateral vocal fold paralysis. Otolaryngol Clin North Am 2012 Oct; 45(5): 1083-1108.

Received: 04/01/2016

Accepted in revised form: 08/02/2016

\section{Corresponding author:}

Mehmet Gökhan Demir, Department of Otorhinolaryngology, Prof. Dr. Celal Ertuğ Etimesgut State Hospital, Ankara, Turkey; email: mgokhandemir@yahoo.com 